\title{
Kidney donation does not increase cardiovascular risk
}

A study conducted in Canada has found no evidence that living kidney donors are at higher risk of cardiovascular events in the first 10 years after donation than are similarly healthy individuals in the general population.

Reduced kidney function is associated with an increased risk of cardiovascular disease in the general population, and it is possible that this increased risk is also present in people who donate a kidney. To investigate, Amit Garg and co-workers conducted a study following living kidney donors in Ontario, Canada, and comparing them with a matched cohort of the healthiest segment of the general population. "We did this study because better knowledge of major cardiovascular events in people who become living kidney donors maintains public trust in the transplantation system, informs the choices of potential donors and recipients, and guides follow-up care to maintain long-term health," state the authors.

Garg et al. used medical charts linked to provincial health-care databases to follow up 2,028 kidney donors and 20,280 matched nondonors (median age 42 years in both groups) for a median of 6.5 years (range 0.1-17.7 years). The primary

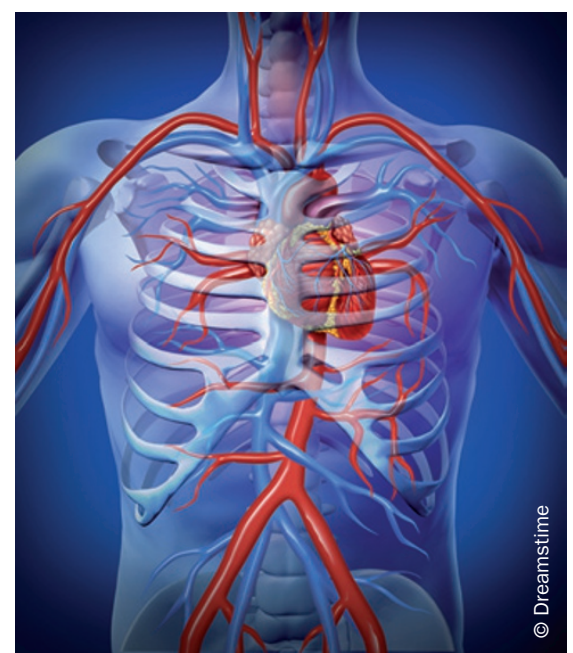

outcome was a composite of time to death or first major cardiovascular event. In total, 609 donors and 5,744 nondonors were followed up for $\geq 10$ years. During follow-up, 381 deaths and 313 major cardiovascular events occurred. The risk of death was lower in donors than in nondonors (2.8 events per 1,000 personyears versus 4.1 events per 1,000 personyears), and there was no difference in the risk of major cardiovascular events censored for death between donors and nondonors ( 1.7 events per 1,000 person-years versus 2.0 events per 1,000 person-years). The researchers found that none of earlier index date, older age at index date, sex, or presence of a first degree relative with kidney failure affected the association between kidney donation and time to death or a cardiovascular event.

"While we will continue to follow up people in this study, these interim results provide important safety reassurances to donors, their recipients, and transplant professionals," note the authors.

"Reassuringly, with longer follow-up the observed risk for the primary outcome continued to be lower in donors than in non-donors. As stated by others, we attribute this lower risk to the rigorous selection process of establishing excellent health before donation, which includes psychological assessment and counselling, abdominal imaging, cancer screening, and an assessment for chronic infectious diseases."

\section{Rebecca Ireland}

Original article Garg, A. X. et al. Cardiovascular disease in kidney donors: matched cohort study. BMJ doi:10.1136/ bmj.e1203 